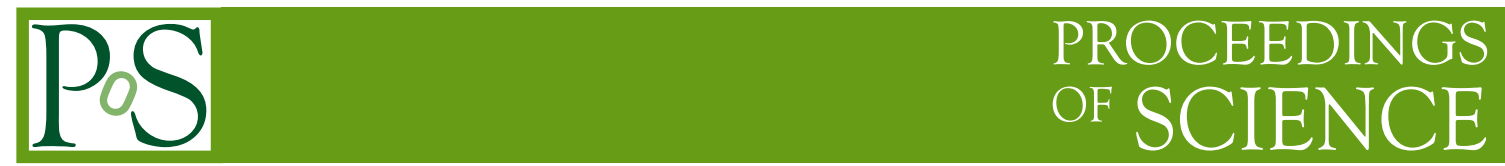

\title{
Measurement of quarkonium production with CMS
}

\author{
Nuno T. Leonardo* \\ On behalf of the CMS Collaboration \\ Purdue University \\ E-mail: nuno.leonardodcern.ch
}

Quarkonium hadroproduction is not satisfactorily understood. Measurements performed at the unprecedented LHC energies, and benefiting from large yields and extended kinematic reach, are anticipated to facilitate crucial improvements to the current understanding. This article presents the initial LHC quarkonium results, based on the data collected by the CMS experiment in the first year LHC pp and $\mathrm{PbPb}$ runs.

The 13th International Conference on B-Physics at Hadron Machines - Beauty2011, April 04-08, 2011

Amsterdam, The Netherlands

\footnotetext{
* Speaker.
} 


\section{Introduction}

Heavy quarkonia constitute an ideal laboratory for testing the interplay between perturbative and nonperturbative quantum chromodynamics (QCD). Considerable progress has been made in establishing effective theories, which rely on both perturbative calculations and lattice simulations. A multitude of data has been accumulated by particle and nuclear physics experiments for more than three decades [1]. The current understanding of quarkonium production remains notwithstanding far from satisfactory.

The Large Hadron Collider (LHC) at CERN is providing sizable datasets of quarkonium decays, which substantially extend the kinematic reach attained by previous experiments. Comprehensive measurements based on these data are expected to contribute significant improvements in our understanding. In the following we summarize the results of measurements of the $\mathrm{J} / \psi$ and $\Upsilon$ families of resonances performed by the Compact Muon Solenoid (CMS) experiment based on the initial 2010 LHC runs.

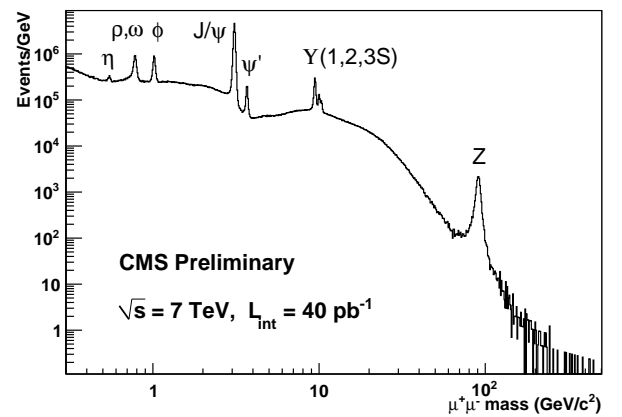

(a) Di-muon mass spectrum, pp@7TeV.

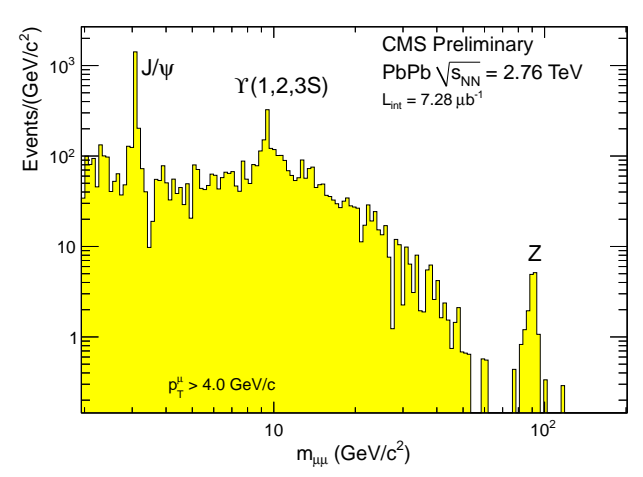

(c) Di-muon mass spectrum, $\mathrm{PbPb} @ 2.36 \mathrm{TeV}$.

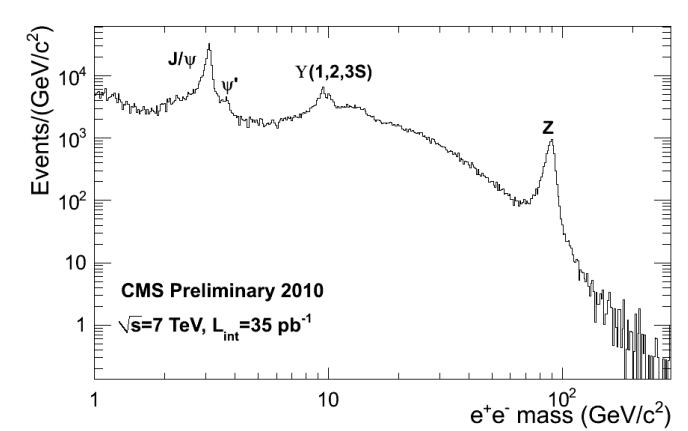

(b) Di-electron mass spectrum, pp@7TeV.

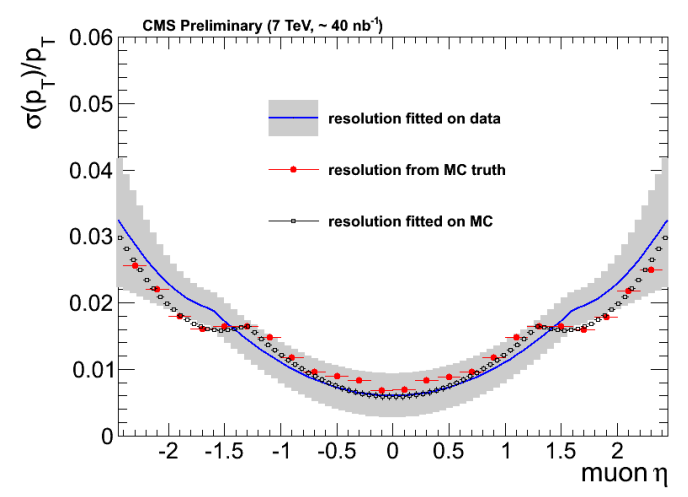

(d) Relative $p_{\mathrm{T}}$ resolution $v s$ muon pseudorapidity, measured in $J / \Psi \rightarrow \mu \mu$ decays in $p p 7 \mathrm{TeV}$ collisions.

Figure 1: The di-lepton mass spectra at CMS, and momentum resolution, from the $2010 \mathrm{pp}$ and $\mathrm{PbPb}$ runs.

\section{Di-lepton signals}

A description of the CMS detector is provided elsewhere [2]. Muons are identified as tracks 
that are reconstructed in the inner silicon tracker which are associated to compatible signals in the outer muon spectrometer [3]. A transverse momentum $\left(p_{\mathrm{T}}\right)$ resolution of about $1 \%$ is attained for muons in the central rapidity region, which degrades to $<6 \%$ in the forward region. The measured relative $p_{\mathrm{T}}$ resolution using $J / \psi$ decays [4] is shown in Fig. 1(d). The CMS dilepton mass spectra are also shown in Fig. 1. Figures 1(a) and 1(b) display the di-muon and di-electron mass distributions, respectively, as measured in $p p$ collisions at $\sqrt{s}=7 \mathrm{TeV}$, while Fig. 1(c) shows the di-muon mass reconstructed in $\mathrm{PbPb}$ collisions at $\sqrt{\mathrm{s}_{\mathrm{NN}}}=2.76 \mathrm{TeV}$. The candidate selection is identical to that employed in the $\Upsilon \rightarrow \mu \mu$ analysis described below, while the di-electron candidates are required to satisfy kinematic $\left(p_{\mathrm{T}}>3 \mathrm{GeV} / c,|\eta|<2\right)$ and isolation $\left(\sum p_{\mathrm{T}}^{\text {track }}<3 \mathrm{GeV} / c\right.$ in a cone $\Delta R<3$ ) criteria. The various known resonant states are prominently observed.

The di-lepton mass distributions in the vicinity of the $J / \psi$ and $\Upsilon(\mathrm{nS})$ mass regions are shown in Figs. 2 and 6 for $p p$ and $\mathrm{PbPb}$ respectively. A di-muon $\mathrm{J} / \psi$ mass resolution of about $25 \mathrm{MeV} / c^{2}$ $\left(50 \mathrm{MeV} / c^{2}\right)$ is attained in the central (forward) detector region, while for the $\Upsilon$ corresponding resolutions of about $70 \mathrm{MeV} / c^{2}\left(140 \mathrm{MeV} / c^{2}\right)$ are achieved. In the di-electron channel, mass resolutions of about $50 \mathrm{MeV} / c^{2}$ and $150 \mathrm{MeV} / c^{2}$ are obtained, for $J / \psi$ and $\Upsilon$ respectively.

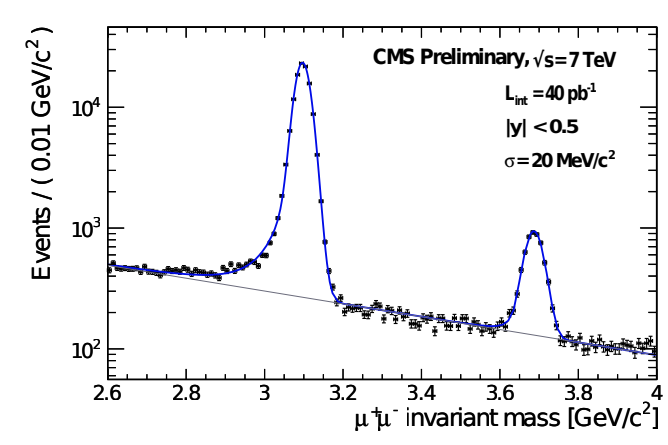

(a) $J / \psi, \psi^{\prime} \rightarrow \mu^{+} \mu^{-}$

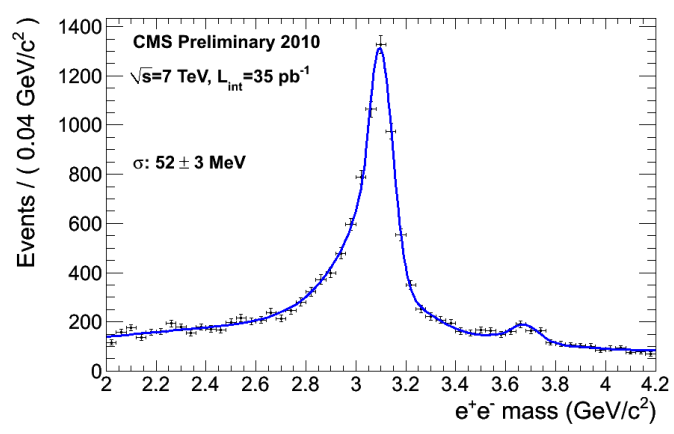

(c) $J / \psi, \psi^{\prime} \rightarrow e^{+} e^{-}$

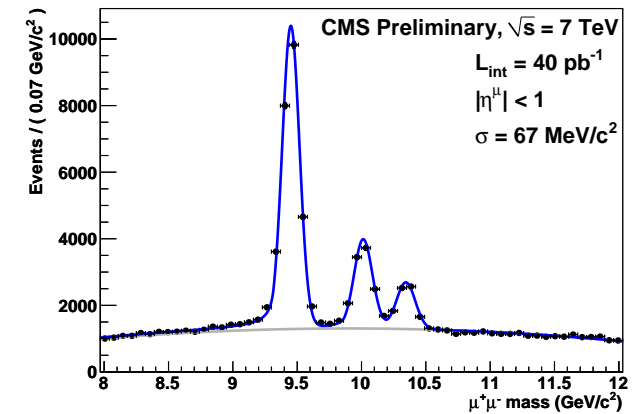

(b) $\Upsilon(1 S, 2 S, 3 S) \rightarrow \mu^{+} \mu^{-}$

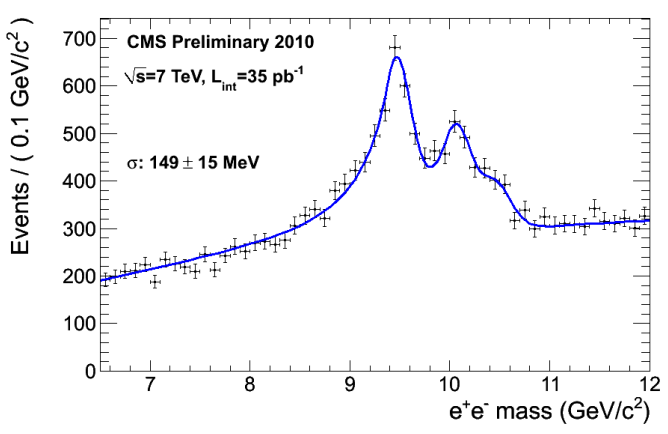

(d) $\Upsilon(1 S, 2 S, 3 S) \rightarrow e^{+} e^{-}$

Figure 2: Di-lepton invariant mass in the vicinity of the $\mathrm{J} / \psi$ and $\Upsilon(\mathrm{nS})$ mass regions.

The study of heavy-quarkonia and, more generally, heavy-flavor production with the initial collision data has primarily explored and relied on di-muon signals. Their online selection is based on the trigger detection of two muons, with no explicit $p_{\mathrm{T}}$ thresholds imposed. 


\section{Charmonium production}

The $J / \psi$ production cross section at $7 \mathrm{TeV}$ is measured employing a dataset of about $0.3 \mathrm{pb}^{-1}$ [5]. Muon tracks are required to pass the following requirements: have at least 12 hits in the Silicon tracker, with at least two of which in the pixel layers; a track fit with a normalized $\chi^{2}$ smaller than four; be closer to the primary vertex than $3 \mathrm{~cm}$ in the transverse direction and $25 \mathrm{~cm}$ along the z-axis. In addition, the muon candidates are required to lie within a kinematic acceptance region defined as: $p_{\mathrm{T}}^{\mu}>3.3 \mathrm{GeV} / c$ for $\left|\eta^{\mu}\right|<1.3$; total momentum $p^{\mu}>2.9 \mathrm{GeV} / c$ for $1.3<\left|\eta^{\mu}\right|<2.2$; and $p_{\mathrm{T}}^{\mu}>0.8 \mathrm{GeV} / c$ for $2.2<\left|\eta^{\mu}\right|<2.4$. An oppositely-charged muon-pair candidate is accepted if its vertex probability is larger than $0.1 \%$. About $27,000 \mathrm{~J} / \psi$ signal candidates are reconstructed.

The differential cross section is experimentally determined from:

$$
\frac{d \sigma}{d p_{\mathrm{T}}}(Q \bar{Q}) \cdot \mathscr{B}\left(Q \bar{Q} \rightarrow \mu^{+} \mu^{-}\right)=\frac{N_{Q \bar{Q}}}{\mathscr{L} \cdot \Delta p_{\mathrm{T}}} \cdot\left\langle\frac{1}{\mathscr{A} \cdot \varepsilon}\right\rangle
$$

where $\mathscr{B}$ is the $Q \bar{Q} \rightarrow \mu \mu$ decay branching fraction, $N_{Q \bar{Q}}$ is the reconstructed quarkonia yield, in a given $p_{\mathrm{T}}$ bin of width $\Delta p_{\mathrm{T}}, \mathscr{L}$ is the integrated luminosity, and $\mathscr{A}$ and $\varepsilon$ are the di-muon acceptance and efficiency.

The geometrical detector acceptance $\mathscr{A}$ is determined from Monte Carlo simulation (MC), by generating signal events in the full kinematical phase space. The quarkonium production polarization strongly influences the muon angular distributions. The current signal statistics is not sufficient to allow for an angular-dependent analysis. Results will be quoted for the unpolarized case. Extreme assumptions for the (not well understood) polarization of prompt quarkonium states result in 20\%-level variations in the average acceptance and, thus, in the final cross-section results.

Muon trigger and reconstruction efficiencies are determined directly from the data, via a "tagand-probe" (T\&P) technique. The $J / \psi \rightarrow \mu \mu$ decay is employed to provide a sample of "probe" muons, by requiring the second decay-leg to be a well-identified muon ("tag"). The tag-probe pairs are divided into two samples, depending on whether the probe satisfies or not the criteria for the efficiency under consideration. The efficiency is extracted from a simultaneous unbinned maximum-likelihood fit to both mass distributions. The quarkonia di-muon efficiency $\varepsilon$ is estimated as the product of single-muon T\&P-determined efficiencies. This factorization assumption is tested using MC and a corresponding systematic uncertainty is assigned. The statistical uncertainties in the muon efficiencies determined with $\mathrm{T} \& \mathrm{P}$ are further propagated as a systematic uncertainty in the cross-section result. This and the uncertainty on the luminosity normalization are the dominant systematic sources on the cross-section determination.

The signal yield is determined via an extended unbinned maximum-likelihood fit to the dimuon mass distribution. The signal shape is parameterized via a template ("Crystal Ball" or CB function) consisting of a Gaussian resolution function with the low-side tail replaced with a power law describing the radiative tail from bremsstrahlung. The shape of the underlying background continuum is described by an exponential. The total cross section for inclusive $J / \psi$ production times branching fraction, for $6.5<p_{\mathrm{T}}<30 \mathrm{GeV} / c$ and rapidity $|y|<2.4$, in the unpolarized production hypothesis, gives $97.5 \pm 1.5$ (stat.) \pm 3.4 (syst.) \pm 10.7 (lumi.) nb. The production $p_{\mathrm{T}}$ spectrum, measured in different rapidity ranges, is shown in Fig. 3(a).

Non-prompt ( $J / \psi$ from $b$-hadron decays) and prompt (including directly produced $J / \psi$ and feed-down from heavier quarkonia states) components are separated using as discriminating vari- 


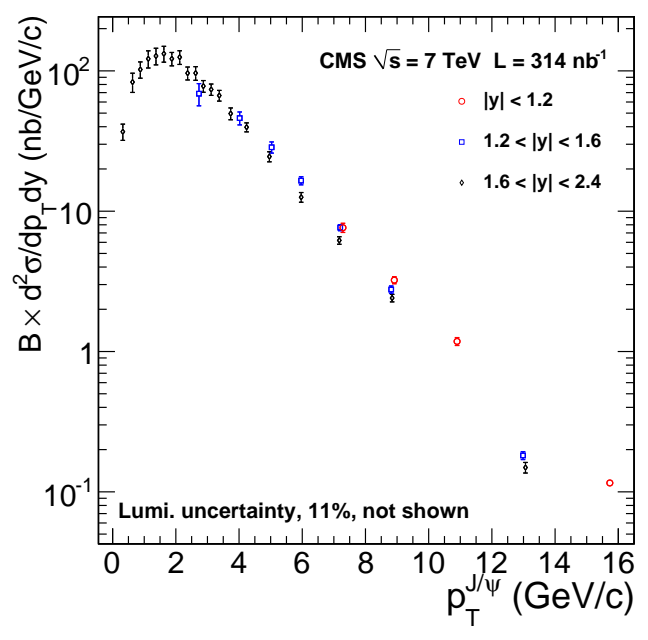

(a) Inclusive $J / \psi$ differential cross section

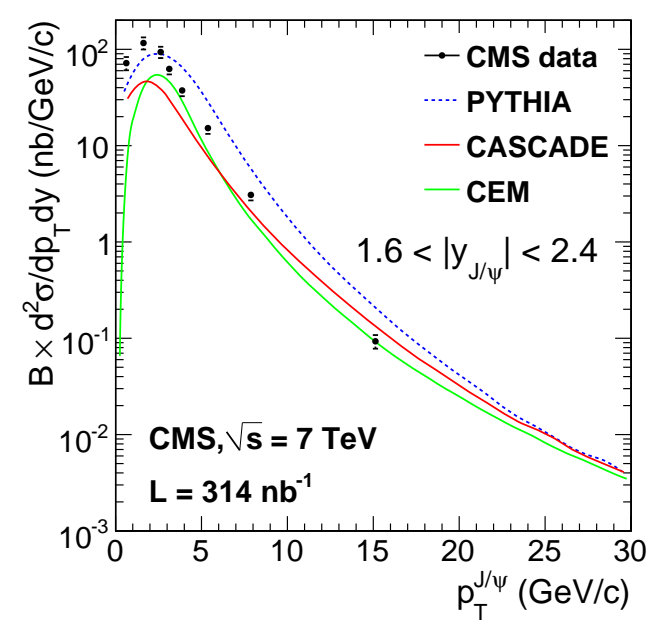

(c) Differential prompt $J / \psi$ cross section

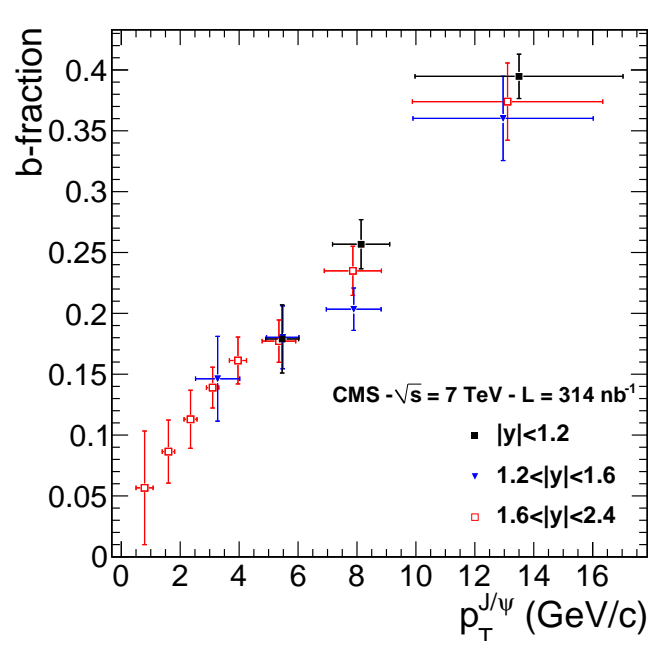

(b) Fraction of $J / \psi$ production from $b$-hadron decays

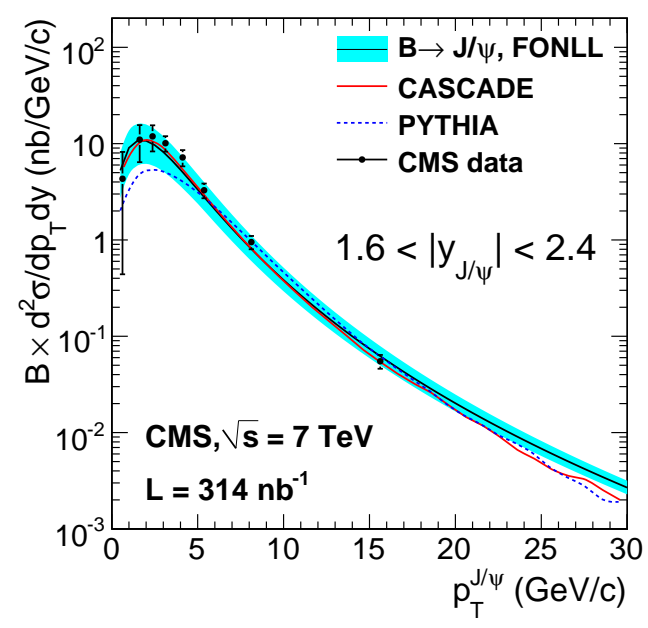

(d) Differential non-prompt $J / \psi$ cross section

Figure 3: Differential $J / \psi$ production cross section and comparison to theory expectations.

able the pseudo-proper decay time, $t$. This is given by $t \equiv L_{x y} \cdot m_{J / \psi} / p_{\mathrm{T}}$, where $m_{J / \psi}$ is the $J / \psi$ mass and $L_{x y}$ is the most probable transverse decay length in the laboratory frame. The fraction of $J / \psi$ from $b$-hadron decays is extracted from a simultaneous fit to the di-muon mass and pseudoproper decay time distributions. It is observed to increase with $p_{\mathrm{T}}$, as seen in Fig. 3(b). This is in turn used for deriving the prompt and non-prompt $J / \psi$ cross sections. In the unpolarized production hypothesis, for $6.5<p_{\mathrm{T}}<30 \mathrm{GeV} / c$ and rapidity $|y|<2.4$, the total cross sections $\sigma \cdot \mathscr{B}$ for prompt and non-prompt $J / \psi$ production are $70.9 \pm 2.1$ (stat.) \pm 3.0 (syst.) \pm 7.8 (lumi.) nb and $26.0 \pm 1.4$ (stat.) \pm 1.6 (syst.) \pm 2.9 (lumi.) nb, respectively. The differential results as a function of $p_{\mathrm{T}}$ for the rapidity range $1.6<|y|<2.4$ are shown in Figs. 3(c) and 3(d). Comparisons to theoretical expectations are also displayed, which show a good agreement for the non-prompt case, whereas for prompt production at low $p_{\mathrm{T}}$ in forward rapidity the predictions underestimate the data. 


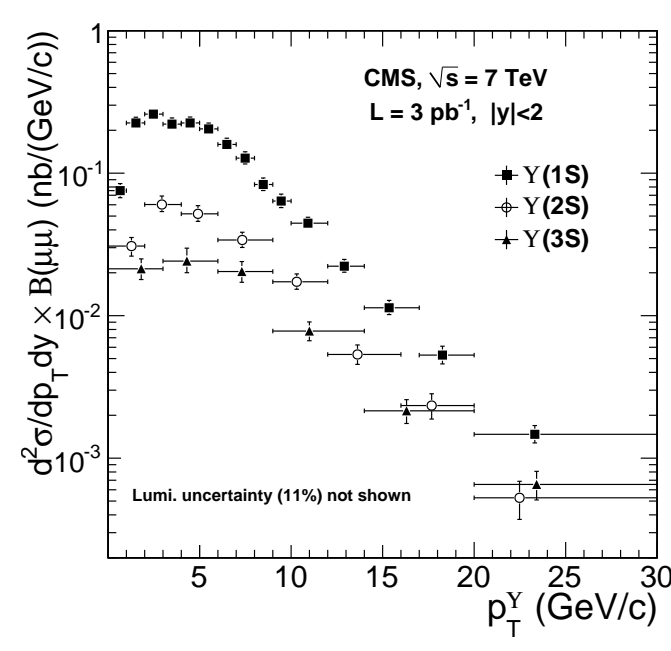

(a) Differential $\Upsilon(\mathrm{nS})$ cross section vs. $p_{\mathrm{T}}$.

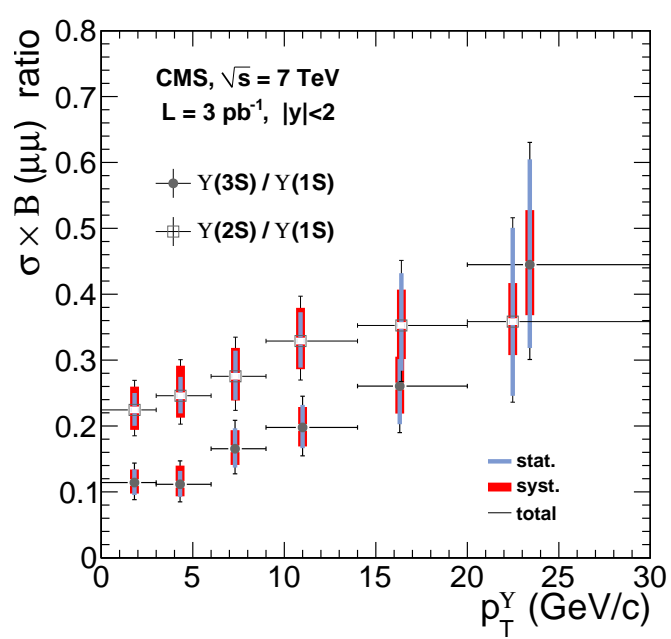

(b) $\Upsilon(\mathrm{nS})$ cross section ratios.

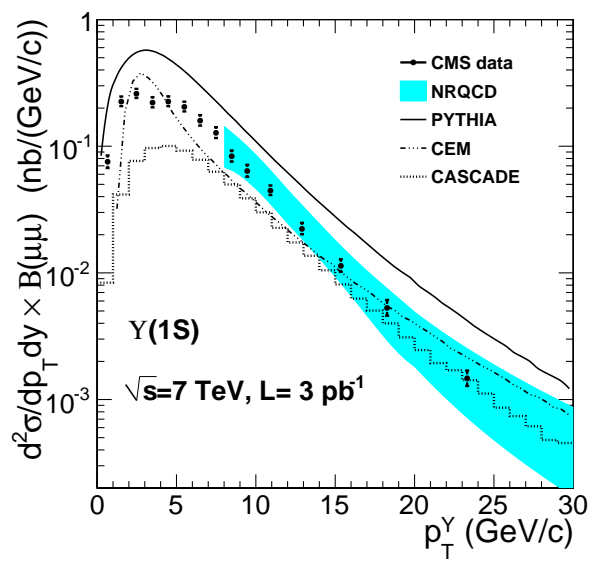

(c) $\Upsilon(1 \mathrm{~S}) d \sigma / d p_{\mathrm{T}}$ and model expectations.

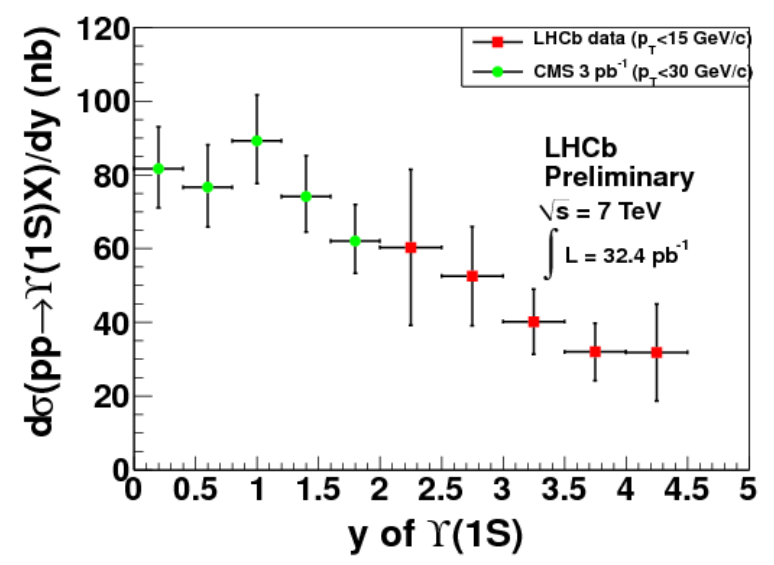

(d) $\Upsilon(1 \mathrm{~S}) d \sigma / d y$, CMS and LHCb rapidity coverage.

Figure 4: Differential $\Upsilon(\mathrm{nS})$ production cross section, comparison to theory expectations and LHCb.

\section{Bottomonium production}

The $\Upsilon(\mathrm{nS})$ production cross section at $7 \mathrm{TeV}$ is measured employing a dataset of about $3 \mathrm{pb}^{-1}$ [6]. The candidate selection is similar to that described in Sec. 3 for the $J / \psi$. Owing to the $\Upsilon$ higher mass, muons from $\Upsilon$ decays have a more uniform detector coverage. A tighter muon kinematic selection is applied: $p_{\mathrm{T}}^{\mu}>3.5 \mathrm{GeV} / c$ for $\left|\eta^{\mu}\right|<1.6$ and $p_{\mathrm{T}}^{\mu}>2.5 \mathrm{GeV} / c$ for $1.6<\left|\eta^{\mu}\right|<2.4$. This reduces the background level while keeping di-muon acceptance down to zero $p_{\mathrm{T}}$ throughout the $\eta$ range.

The corrected signal yields are extracted directly from an unbinned extended maximum-likelihood fit to the di-muon mass. The mass line-shape for each of the $\Upsilon(\mathrm{nS})$ states is given by a CB function, while the background is empirically modeled via a second-order polynomial. The acceptance and efficiency corrections are assigned on a candidate-by-candidate basis, based on its measured kinematic properties, via an event weight given by $1 /(\mathscr{A} \cdot \varepsilon)$. That is, the cross section is determined as 


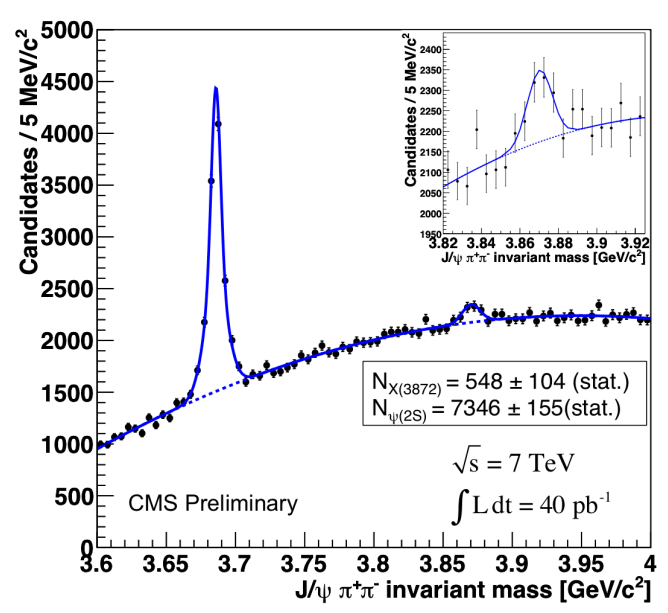

(a) $\mu^{+} \mu^{-} \pi^{+} \pi^{-}$invariant mass, showing the $\psi^{\prime}$ and $\mathrm{X}(3872)$ decays to $J / \psi \pi \pi$

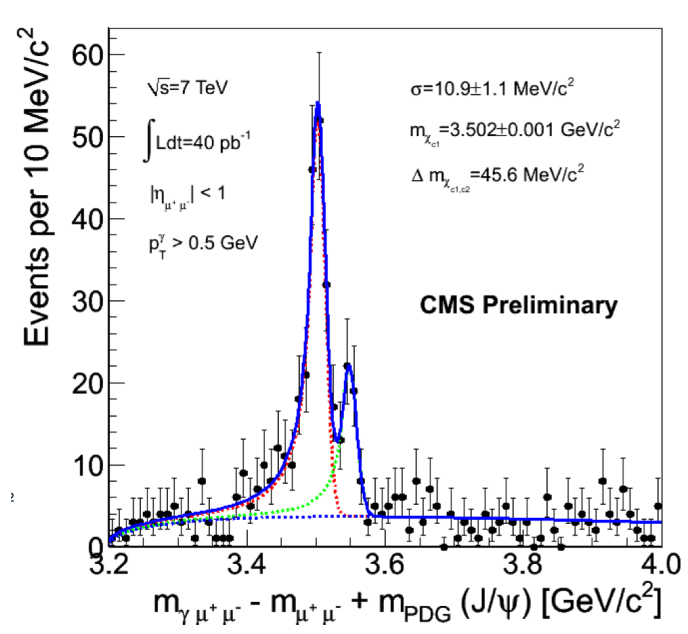

(b) $\mu^{+} \mu^{-} \gamma$ and $\mu^{+} \mu^{-}$invariant mass differences, showing the $\chi_{c 1}$ and $\chi_{c 2}$ decays to $J / \psi \gamma$

Figure 5: Observation of the $\mathrm{X}(3872)$ and $\chi_{c}$ states in the $40 \mathrm{pb}^{-1}$ at $7 \mathrm{TeV}$ pp dataset.

in Eq. 3.1, with the r.h.s. evaluated on a per-event basis instead of averaged over kinematic ranges.

The measured total cross-sections, for the rapidity range $|y|<2$ and assuming unpolarized production, are $7.37 \pm 0.13$ (stat. $)_{-0.42}^{+0.61}$ (syst.) \pm 0.81 (lumi.) nb, $1.90 \pm 0.08$ (stat. $)_{-0.12}^{+0.18}$ (syst.) \pm 0.21 (lumi.) nb, $1.02 \pm 0.07$ (stat. $)_{-0.08}^{+0.11}$ (syst.) \pm 0.11 (lumi.) nb, for the $\Upsilon(1 \mathrm{~S}), \Upsilon(2 \mathrm{~S})$, and $\Upsilon(3 \mathrm{~S})$ states respectively. The differential results as a function of $p_{\mathrm{T}}$ are shown in Fig. 4(a) The ratios of cross sections of the excited states $(2 \mathrm{~S}, 3 \mathrm{~S})$ relative to the ground state (1S) are observed in Fig. 4(b) to increase with $p_{\mathrm{T}}$.

Comparisons with model predictions are shown in Fig. 4(c). The predicted PYTHIA shape agrees with the data, while the normalization is overestimated by a factor of two. The NRQCD expectation agrees with the measured spectrum for $p_{\mathrm{T}}>8 \mathrm{GeV} / c$. The model predictions are however preliminary, with some currently lacking uncertainty estimations. The results are further compared with other measurements. A good agreement of the normalized $p_{\mathrm{T}}$-differential cross sections is obtained with the previous Tevatron measurements at smaller collision energies, while the integrated cross section is about a factor of three larger at the LHC. The rapidity-differential cross section for the $\Upsilon(1 \mathrm{~S})$ state is shown in Fig. 4(d). It also shows a comparison with preliminary LHCb results [7] at extended forward rapidities, nicely displaying the complementarity of the two experiments in terms of phase-space coverage.

\section{5. $\mathbf{X}(3872)$ and $\psi^{\prime}$ cross-section ratio}

The $\mathrm{X}(3872)$ state is the firstly observed and most intriguing of a set of unanticipated ("exotic") states. It was discovered by Belle in 2003, in $B^{+} \rightarrow J / \psi K^{+} \pi^{+} \pi^{-}$decays as a narrow peak in the $J / \psi \pi \pi$ final state mass, and it has since been widely studied at the $B$-factories and the Tevatron. It has a mass of $3871.6 \mathrm{MeV} / c^{2}$, a width of $2.3 \mathrm{MeV} / c^{2}$ (90\% CL upper limit), and two possible $J^{P C}$ assignments $1^{++}$and $2^{-+}$. Its interpretation however is not established - proposed models include 
molecular $D^{0} \bar{D}^{\star 0}-D^{* 0} \overline{D^{0}}$ bound state, diquark-antidiquark, $c \bar{c} g$ hybrid meson - and demands further scrutiny. A recent summary of the theoretical and experimental status is provided in Ref. [1].

The $\mathrm{X}(3872) \rightarrow J / \psi \pi^{+} \pi^{-}$signal is observed and analyzed employing a $40 \mathrm{pb}^{-1}$ dataset [8]. The muon selection is similar to that presented in Sec. 3. Track-quality and selection criteria are applied to pion track candidates satisfying $p_{\mathrm{T}}>1.5 \mathrm{GeV} / c$. The oppositely charged muon pair (constrained to the $J / \psi$ mass) and pion pair are combined, required to be within an opening angle $\Delta R<0.7$, and the four tracks are refitted assuming origin from a common vertex. The invariantmass distribution is shown in Fig. 5(a) where clear signals of the $\mathrm{X}$ and $\psi^{\prime}$ are observed. These are fit with single and double Gaussian models, respectively, yielding about $500 \mathrm{X}$ and $7000 \psi^{\prime}$.

The $\mathrm{X}(3872)$ yield, corrected for efficiency and acceptance, is determined relative to the corrected yield of $\psi^{\prime}$ mesons decaying to the same final state, in the kinematic region $p_{\mathrm{T}}>8 \mathrm{GeV} / \mathrm{c}$ and $|y|<2.2$. The measured $X / \psi^{\prime}$ cross-section ratio is $0.087 \pm 0.017$ (stat.) \pm 0.009 (syst.). The ratio has the advantage that systematic uncertainties related to triggering and reconstruction of $J / \psi$, as well as normalization uncertainties, such as from the integrated luminosity, largely cancel.

\section{6. $\chi_{c}$ states}

Prompt quarkonium production incorporates directly produced quarkonia as well as feed-down from higher-mass states. In fact, a substantial fraction, of about 30-50\%, of the quarkonium ground states $J / \psi$ and $\Upsilon(1 \mathrm{~S})$ produced in hadronic collisions originate from the decay of higher excitations [1]. Such components are not disentangled in inclusive measurements, such as reported in Sections 3 and 4. There are however many unresolved theoretical issues at present that bear on the reliability of predictions for prompt production, and these are expected to greatly benefit from measurements where direct production of $J / \psi$ and $\Upsilon$ is separated from production via feed-down from higher-mass charmonium and bottomonium states. This is of relevance, too, for the forthcoming measurements of quarkonium polarization (or spin-alignment, via its decay angular analysis).

Figure 5(b) shows the observation of the charmonium states $\chi_{c 1}$ and $\chi_{c 2}$ through the radiative decay to $J / \psi \gamma$, using a $40 \mathrm{pb}^{-1}$ dataset [9]. Photons are reconstructed using a $\gamma$-conversion reconstruction technique. The signal lineshapes are described by CB functions, and the mass difference is fixed in the fit. A $\chi_{c 1}$ mass of $3.502 \pm 0.001 \mathrm{GeV} / c^{2}$ and a resolution of $11 \pm 1 \mathrm{MeV} / c^{2}$ are obtained.

\section{Quarkonia in heavy-ion collisions}

At large energy densities and/or high temperatures, strongly-interacting matter is predicted, and demonstrated using lattice QCD simulations, to undergo a phase transition to a new state where deconfinement of quarks and gluons should set in. Characterization of the properties of this form of matter, referred to as quark-gluon plasma (QGP), constitutes the main objective of studies performed with relativistic heavy-ion collisions. Heavy quarkonia are considered one of the most promising probes of the formed medium. Indeed, Debye screening of the confining potential of heavy quark-antiquark pairs, and consequent suppression of charmonium and bottomonium states, sequentially in order of binding energy, may provide an important signature of deconfinement. 


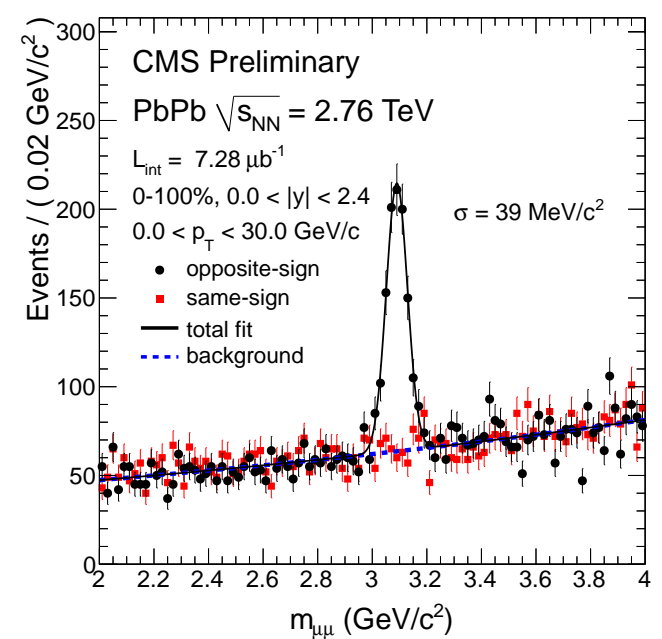

(a) $J / \psi$ mass region.

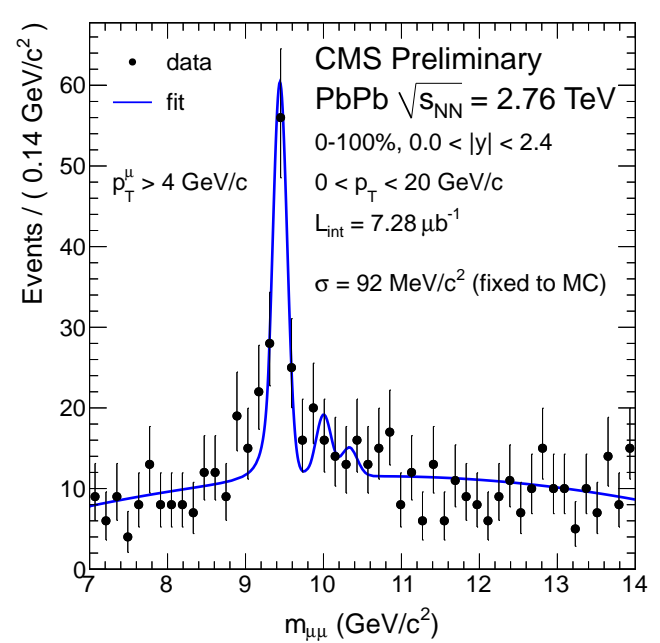

(b) $\Upsilon(\mathrm{nS})$ mass region.

Figure 6: Di-muon invariant mass in $\mathrm{PbPb}$ collisions at $\sqrt{\mathrm{S}_{\mathrm{NN}}}=2.76 \mathrm{TeV}$.

The higher energy and luminosity available in heavy-ion collisions at the LHC allow studies of charmonia, in a different energy regime than at SPS and RHIC, and first significant measurements of the bottomonium family.

CMS has collected $\mathrm{a} \sim 7 \mu \mathrm{b}^{-1}$ dataset of $\mathrm{PbPb}$ collisions at a centre-of-mass nucleon-nucleon energy of $\sqrt{\mathrm{s}_{\mathrm{NN}}}=2.76 \mathrm{TeV}$ from the heavy-ion LHC run at the end of 2010. A stricter trackreconstruction algorithm than that used in $p p$, optimized for the higher event multiplicity, is employed. Similar trigger selection and $\eta$-dependent $p_{\mathrm{T}}$ muon thresholds are adopted; in the case of the $\Upsilon$ analysis a stricter cut $p_{\mathrm{T}}^{\mu}>4 \mathrm{GeV} / c$ is applied. The measured di-muon mass distributions are shown in Fig. 6. About $700 J / \psi$ and $100 \Upsilon$ are observed. The $J / \psi$ and $\Upsilon(1 S)$ invariant yields are determined [10] following an analysis approach similar to what is described in Sections 3 and 4. Differential measurements are performed as a function of $p_{\mathrm{T}}$, rapidity, and centrality of the collision. Separation of prompt and non-prompt $J / \psi$ and of the distinct $\Upsilon(\mathrm{nS})$ states is performed for the first time in the heavy-ion environment. Comparison of figures 2(b) and 6(b) hints a suppression of the excited states relative to the ground $\Upsilon$ state [11], a signature compatible with QGP formation.

\section{Summary}

The first-year run of the LHC has been explored by the CMS experiment to deliver a significant first set of quarkonium production results in both proton-proton and heavy-ion environments.

\section{References}

[1] N. Brambilla et al., CERN-2005-005 (2004), arXiv:hep-ph/0412158v2; Eur. Phys. J. C 71 (2011) 1534, arXiv: 1010.5827 [hep-ph]

[3] CMS Coll., CMS-PAS-MUO-10-002 (2010)

[4] CMS Coll., CMS-PAS-TRK-10-004 (2010)

[2] CMS Coll., JINST 0803:S08004 (2008)

[5] CMS Coll., Eur.Phys.J.C71:1575 (2010) 
[6] CMS Coll., CMS-BPH-10-003 (2010)

[7] LHCb Coll., LHCb-ANA-2011-012 (2011)

[8] CMS Coll., CMS-PAS-BPH-10-018 (2011)
[9] CMS Coll., CMS-DP-2011-006 (2011)

[10] CMS Coll., CMS-PAS-HIN-10-006 (2011)

[11] CMS Coll., CMS-HIN-11-007 (2011) 